\title{
Prevalence and mechanisms of cortical superficial siderosis in cerebral amyloid angiopathy \\ OPEN
}

Andreas Charidimou, MSc

Rolf Hans Jäger, MD

Zoe Fox, PhD

Andre Peeters, MD

Yves Vandermeeren, PhD

Patrice Laloux, PhD

Jean-Claude Baron, PhD

David John Werring,

$\mathrm{PhD}$

Correspondence to

Dr. Werring:

d.werring@ucl.ac.uk

Supplemental data at www.neurology.org

\section{ABSTRACT}

Objective: We investigated the prevalence and clinical-radiologic associations of cortical superficial siderosis (CSS) in patients with probable cerebral amyloid angiopathy (CAA) compared to those with intracerebral hemorrhage $(\mathrm{ICH})$ not attributed to CAA.

Methods: We conducted a retrospective multicenter cohort study of 120 patients with probable CAA and 2 comparison groups: 67 patients with either single lobar ICH or mixed (deep and lobar) hemorrhages; and 22 patients with strictly deep hemorrhages. We rated cSS, ICH, white matter changes, and cerebral microbleeds.

Results: cSS was detected in 48 of 120 (40\%; 95\% confidence interval [Cl]: 31.2\%-49.3\%) patients with probable CAA, 10 of 67 (14.9\%; 95\% Cl: 7.4\%-25.7\%) with single lobar ICH or mixed hemorrhages, and 1 of 22 ( $4.6 \%$; $95 \% \mathrm{Cl}: 0.1 \%-22.8 \%)$ patients with strictly deep hemorrhages $(p<0.001$ for trend). Disseminated cSS was present in 29 of 120 (24\%; 95\% Cl: 16.8\%-32.8\%) patients with probable CAA, but none of the other patients with $\mathrm{ICH}(\mathrm{p}<0.001)$. In probable $\mathrm{CAA}$, age (odds ratio [OR]: 1.09; 95\% Cl: 1.03-1.15; $p=0.002)$, chronic lobar ICH (OR: 3.94; 95\% Cl: 1.54-10.08; $p=$ 0.004 ), and a history of transient focal neurologic episodes (OR: 11.08; 95\% Cl: 3.49-35.19; $p<$ 0.001 ) were independently associated with cSS. However, cSS occurred in 17 of 48 patients with probable CAA (35.4\%; 95\% Cl: 22.2\%-50.5\%) without chronic lobar $\mathrm{ICH}$.

Conclusions: cSS (particularly if disseminated) is a common and characteristic feature of CAA. Chronic lobar ICH is an independent risk factor for cSS, but the causal direction and mechanism of association are uncertain. Hemorrhage into the subarachnoid space, independent of previous (chronic) lobar ICH, must also contribute to cSS in CAA. Transient focal neurologic episodes are the strongest clinical marker of cSS. Neurology ${ }^{\circledR}$ 2013;81:626-632

\section{GLOSSARY}

CAA = cerebral amyloid angiopathy; $\mathbf{C I}=$ confidence interval; $\mathbf{C M B}=$ cerebral microbleed; $\mathbf{C S A H}=$ convexity subarachnoid hemorrhage; $\mathbf{c S S}$ = cortical superficial siderosis; FLAIR = fluid-attenuated inversion recovery; $\mathbf{I C H}=$ intracerebral hemorrhage; $\mathbf{O R}=$ odds ratio; T2*-GRE $=\mathrm{T2}^{*}$-weighted gradient recalled echo.

Sporadic cerebral amyloid angiopathy (CAA) is a common age-related small-vessel disease caused by progressive deposition of amyloid- $\beta$ in the walls of small arteries, arterioles, and capillaries in the cerebral cortex and overlying leptomeninges. ${ }^{1} \mathrm{CAA}$ is most often recognized in life by symptomatic lobar intracerebral hemorrhage (ICH) in elderly patients. ${ }^{1-3}$ CAA is also associated with characteristic MRI findings including lobar cerebral microbleeds (CMBs) and white matter hyperintensities (leukoaraiosis). ${ }^{1,4}$

Recent studies have identified cortical superficial siderosis (cSS) as another manifestation of $\mathrm{CAA}^{5-8}$ In CAA, cSS has a characteristic predilection for the cerebral convexities, reflecting linear blood residues in the superficial layers of the cerebral cortex or in the subarachnoid space. ${ }^{8-10} \mathrm{cSS}$ may

\footnotetext{
From the Stroke Research Group, Department of Brain Repair and Rehabilitation (A.C., D.J.W.), Lysholm Department of Neuroradiology (R.H.J.), UCL Institute of Neurology and The National Hospital for Neurology and Neurosurgery; Department of Brain Repair and Rehabilitation (R.H.J.), Biomedical Research Centre (Z.F.), UCL and the Education Unit (Z.F.), UCL Institute of Neurology, Queen Square, London, UK; Department of Neurology (A.P.), Cliniques Universitaires UCL Saint Luc, Brussels; Department of Neurology, CHU Mont-Godinne (Y.V., P.L.), Institute of Neuroscience (Y.V., P.L.), Université Catholique de Louvain, Brussels, Belgium; Department of Clinical Neurosciences (J.-C.C.B.), University of Cambridge, Addenbrooke's Hospital, Cambridge, UK; and Inserm U894 (J.-C.B.), Centre Hospitalier Sainte-Anne, Sorbonne Paris Cité, Paris, France. Go to Neurology.org for full disclosures. Funding information and disclosures deemed relevant by the authors, if any, are provided at the end of the article. This is an open access article distributed under the Creative Commons Attribution License, which permits unrestricted use, distribution, and reproduction in any medium, provided the original work is properly cited.
} 
have clinical relevance as an important cause of transient focal neurologic episodes (sometimes called "amyloid spells"), ${ }^{11,12}$ and a potential "warning sign" for future symptomatic ICH. ${ }^{13}$

Although cSS is a promising diagnostic neuroimaging marker of $\mathrm{CAA},{ }^{8}$ the strength of the association and underlying mechanisms have not been systematically studied. In the present study, we therefore sought to determine the prevalence and extent of cSS in a European multicenter cohort of patients with clinical-radiologic probable CAA, and investigate its associations with other imaging findings including CMBs, white matter changes, and $\mathrm{ICH}$. We hypothesized that cSS 1) is common in subjects with probable CAA, and more prevalent compared to comparison subjects with other spontaneous $\mathrm{ICH}$ not attributed to probable CAA, and 2) is associated with other hemorrhagic manifestations of CAA (lobar ICH and lobar CMBs).

METHODS Study population. We included consecutive patients diagnosed with probable CAA (according to the original Boston criteria, ${ }^{14}$ i.e., not including cSS as a criterion; see below) at 4 stroke centers over defined time periods (figure e- 1 on the Neurology ${ }^{8}$ Web site at www.neurology.org). At participating centers, MRI scanning is a routine investigation for cases of suspected CAA, unless there are contraindications. Essential inclusion criteria for the main CAA case group were 1) probable or pathologically verified CAA, defined according to the Boston criteria, ${ }^{14}$ including lobar CMBs, but not cSS; and 2) available MRI sequences of adequate quality including T2*-weighted gradient recalled echo (T2*-GRE) and fluid-attenuated inversion recovery (FLAIR) MRI sequences. Cases defined as possible CAA according to the Boston criteria ${ }^{14}$ (i.e., single lobar ICH without lobar microbleeds) were included in one of the comparison groups (see next paragraph).

As comparison groups, we included patients with other symptomatic, spontaneous ICH not fulfilling the Boston criteria for probable CAA, seen in the 4 stroke centers over the same time period, with available MRI scans of adequate quality including T2*-GRE and FLAIR sequences. Cases were systematically and consecutively ascertained using multiple overlapping methods from prospective clinical and radiologic databases. The 2 non-CAA comparison groups were as follows: a) single lobar ICH (without any CMBs) and mixed (deep and lobar) hemorrhages (including any combination of ICH or $\mathrm{CMBs}$ ), which we subsequently refer to as "single lobar ICH and mixed hemorrhages"; and b) strictly deep hemorrhages (including $\mathrm{ICH}$ and $\mathrm{CMBs}$ in the basal ganglia and brainstem).

In total, 149 subjects were excluded from our study because MRI scans were unavailable (figure e-1); those excluded were not significantly different from those included in sex, age, or measures of stroke severity (all $p$ values $>0.10$; figure e- 1 ).

Standard protocol approvals, registrations, and patient consents. The study received ethical approval by the National Hospital for Neurology and Neurosurgery and Institute of Neurology Joint Research Ethics Committee, the Commission d'Ethique Biomedicale Hospitalo Facultaire of the Faculte de Medicine (Universite Catholique de Louvain), and the Comite d'ethique medicale of the CHU Mont Godinne UCL.
Data collection. Demographic and clinical information was obtained from prospective databases and by medical records review using standardized data collection forms. Hypertension was defined as a history of hypertension, taking antihypertensive treatment, or documented elevated blood pressure (systolic $>150$ or diastolic $>95 \mathrm{~mm} \mathrm{Hg}$ ) before admission; diabetes as ongoing use of a hypoglycemic agent; and smoking as history of tobacco use before admission. In patients with probable CAA, a clearly documented history of transient $(\leq 24$ hours), fully resolving, focal neurologic episodes with no known alternative explanation other than CAA (e.g., structural brain lesion, atrial fibrillation, or extracranial or intracranial stenosis) was ascertained by review of medical records.

MRI acquisition and analysis. The MRI protocol was similar in each hospital. Imaging was at 1.5 -tesla field strength for all patients and included T1-weighted, T2-weighted, FLAIR, T2*-GRE (slice thickness $5 \mathrm{~mm}$, repetition time 500-1,000 milliseconds, echo time 40/26/15/50-70 milliseconds), and diffusion-weighted imaging sequences. Images were reviewed blinded to clinical data. Hemorrhagic lesions, ${ }^{4,8,15,16}$ ischemic lesions (chronic or acute), ${ }^{17}$ and white matter hyperintensities (leukoaraiosis) ${ }^{18}$ were recorded according to predefined standardized criteria. CMBs were evaluated on T2*-GRE images. ${ }^{4,16}$ Prior symptomatic ICH was defined as a symptomatic stroke syndrome associated with imaging evidence of a corresponding "macro" $\mathrm{ICH}\left(>5 \mathrm{~mm}\right.$ in diameter on T2*-GRE). ${ }^{15}$ Asymptomatic prior ICH ( $>5 \mathrm{~mm}$ in diameter on T2*-GRE MRI) was also noted: chronic ICH was defined on neuroimaging as ICH with no acute bleeding identified on either CT or MRI scans. Acute convexity subarachnoid hemorrhage $(\mathrm{CSAH})$ was defined as linear hypointensity in the subarachnoid space affecting one or more cortical sulci of the cerebral convexities on T2*GRE sequences with corresponding hyperintensity in the subarachnoid space on T1-weighted or FLAIR images. cSS was defined as linear residues of chronic blood products in the superficial layers of the cerebral cortex showing a characteristic "gyriform" pattern of low signal on T2*-GRE images, without corresponding hyperintense signal on T1-weighted or FLAIR images. We did not include cSS if it was contiguous with any ICH. The distribution of CSS and acute $\mathrm{CSAH}$ was classified as focal (restricted to $\leq 3$ sulci) or disseminated ( $\geq 4$ sulci). ${ }^{8}$ We also noted whether cSS was contralateral or ipsilateral to any chronic ICH. Using a sample of MRI scans from patients with probable CAA $(n=48)$ and control, non-CAA cases $(n=27)$, the interrater agreement for the presence or absence of cSS was 93.3\% (Cohen $\kappa=0.85$ ) and for cSS categories was $95.1 \%$ (weighted Cohen $\kappa=0.86$ ). White matter hyperintensities (leukoaraiosis) were assessed with the 4-step simplified Fazekas Rating Scale, from 0 to 3 ( $0=$ no lesions; 1 = focal lesions; 2 = early confluent; 3 = confluent) ${ }^{18}$

Statistical analysis. We compared demographic, clinical, and imaging characteristics of patients with probable CAA, with vs without cSS. Both binary and ordinal logistic regression analyses were used to identify predictors of cSS (cSS presence and cSS extent, respectively). Multivariable analysis was adjusted for age, lobar $\mathrm{CMB}$ (used as a categorical variable: $0,1,2-4$, and $>4$ $\mathrm{CMBs}$ ), presence of chronic lobar $\mathrm{ICH}$, and history of transient focal neurologic episodes, based on the results of univariable analysis, plus other biologically plausible confounders. As a sensitivity analysis, we repeated these analyses with the addition of hypertension in our multivariable regression models. Because the results of binary and ordinal logistic regression analyses were consistent, we present only the results of the binary logistic regression analysis. A $p$ value $\leq 0.05$ was considered to be statistically significant. Data were missing in less than $5 \%$ of participants; these cases were excluded from univariable and multivariable analyses that included a missing variable of interest. For the main multivariable model presented, there were no missing data. All statistical 


\begin{tabular}{|c|c|c|c|}
\hline \multicolumn{4}{|c|}{$\begin{array}{l}\text { Characteristics of patients with probable CAA and our comparison patient groups with spontaneous } \\
\text { symptomatic ICH not fulfilling the Boston criteria for probable CAA: "Single lobar ICH and mixed } \\
\text { hemorrhages" and "strictly deep hemorrhages"a }\end{array}$} \\
\hline & & Comparison groups not fulfilling & teria for probable CAA \\
\hline Characteristics & Probable CAA $(n=120)$ & $\begin{array}{l}\text { Single lobar } \mathrm{ICH} / \text { mixed deep } \\
\text { and lobar hemorrhages }(n=67)\end{array}$ & $\begin{array}{l}\text { Strictly deep } \\
\text { hemorrhages }(n=22)\end{array}$ \\
\hline Age, y & $71.8(70-73.5)$ & $72.6(61.3-75.3)$ & $61.2(58.5-71.8)$ \\
\hline Male & $67(55.8)$ & 35 (52.3) & 15 (68.2) \\
\hline Hypertension & $72(64.3)$ & 48 (71.6) & 21 (99.5) \\
\hline Taking antithrombotics & 25 (22.1) & 24 (35.8) & 7 (31.8) \\
\hline Any symptomatic ICH & $104(86.7)^{b}$ & $67(100)$ & $22(100)$ \\
\hline Cortical superficial siderosis & $48(40)$ & 10 (14.9) & $1(4.6)$ \\
\hline Focal, $\leq 3$ sulci & 19 (15.8) & $10(14.9)$ & $1(4.6)$ \\
\hline Disseminated, $\geq 4$ sulci & 29 (24.2) & $0(0)$ & $0(0)$ \\
\hline Acute cSAH & 15 (12.5) & $3(4)$ & $0(0)$ \\
\hline
\end{tabular}

Abbreviations: $\mathrm{CAA}=$ cerebral amyloid angiopathy; $\mathrm{CSAH}=$ convexity subarachnoid hemorrhage; $\mathrm{ICH}=$ intracerebral hemorrhage.

${ }^{a}$ Data are mean (95\% confidence interval) or $\mathrm{n}(\%)$.

${ }^{b}$ One hundred four of the patients with probable CAA were admitted for spontaneous lobar ICH, 8 with transient focal neurologic episodes, 2 because of cognitive decline, 2 with acute $\mathrm{CSAH}$, and 2 because of an ischemic stroke.

analyses were performed using STATA software (version 11.2; StataCorp, College Station, TX).

RESULTS The final cohort consisted of 209 patients: 120 patients with probable CAA (9 with supportive pathology) based on the Boston criteria and 89 patients with other ICH not fulfilling the Boston criteria for probable CAA, forming 2 comparison groups- $67 \mathrm{pa}-$ tients with a single lobar ICH or mixed (lobar and

Figure 1 Prevalence $(\%, 95 \% \mathrm{Cl})$ of cSS in different groups

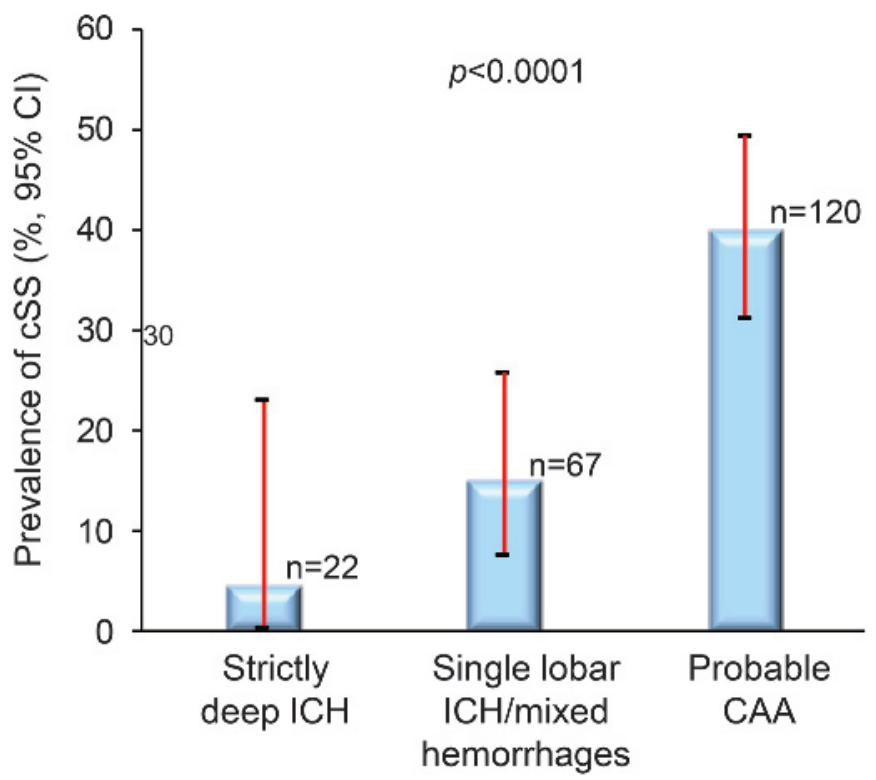

The prevalence of cortical superficial siderosis (cSS) is significantly higher in patients with probable cerebral amyloid angiopathy (CAA) compared with the comparison groups (single lobar intracerebral hemorrhage $[\mathrm{ICH}]$ and mixed hemorrhages, and strictly deep hemorrhages). The $p$ value for trend calculated using $x^{2}$ test. $\mathrm{Cl}=$ confidence interval. deep) hemorrhages, and 22 patients with strictly deep hemorrhages (table 1).

cSS was detected in 48 of 120 (40\%; 95\% confidence interval $[\mathrm{CI}]: 31.2 \%-49.3 \%)$ patients with probable CAA, compared with 10 of 67 (14.9\%; 95\% CI: $7.4 \%-25.7 \%$ ) of the patients with a single lobar ICH or mixed (lobar and deep) hemorrhages, and only 1 of 22 (4.6\%) patients with strictly deep hemorrhages $(p<$ 0.001 for trend) (table 1 and figure 1). Within the single lobar ICH or mixed hemorrhage group, cSS was found in 2 of $33(6.1 \%)$ patients with single lobar ICH and no CMBs, 2 of 11 (18\%) patients with lobar ICH and deep or mixed CMBs, and 3 of 17 (18\%) patients with deep $\mathrm{ICH}$ and lobar or mixed $\mathrm{CMBs}$ (see table e- 1 for the frequency of cSS in detailed patient subgroups). Disseminated cSS was observed in 29 of 120 (24.2\%; 95\% CI: $16.8 \%-32.8 \%$ ) patients with probable CAA but in none of the other 89 patients not fulfilling the original Boston criteria for probable CAA $(p<0.001)$. In patients with single lobar ICH or mixed hemorrhages, cSS (10 of 67) was always focal; of these 10 patients with cSS, 7 (70\%) had lobar CMBs (table e-2).

Thirty-one of the 48 patients with probable CAA and cSS had chronic lobar ICH; the cSS was contralateral to the ICH or bilateral in $24(87.4 \%)$ of these patients and restricted to the hemisphere ipsilateral to chronic lobar $\mathrm{ICH}$ in only 7 patients (22.6\%) (i.e., in most cases, cSS was present distant from any chronic lobar ICH). In 17 of the 48 patients with probable CAA and cSS (35\%), there was no evidence of chronic lobar ICH. Representative examples of cSS are shown in figure 2.

Within the probable CAA group, patients with cSS were older and less often hypertensive compared 
Figure 2 T2*-GRE and FLAIR MRIs of 2 patients with probable CAA and a patient with mixed deep and lobar hemorrhages
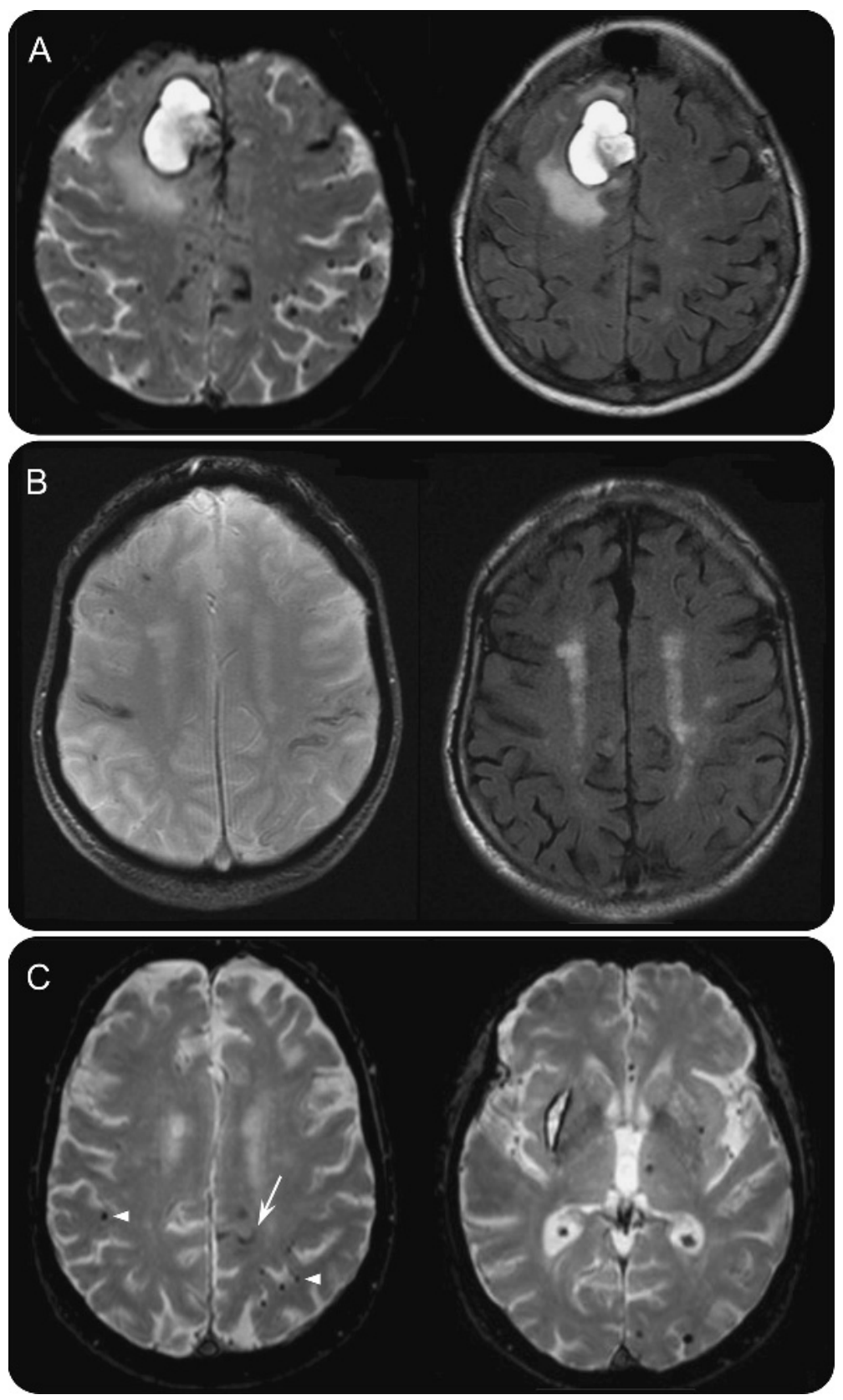

(A) Patient with probable cerebral amyloid angiopathy (CAA) and an acute right frontal intracerebral hemorrhage $(\mathrm{ICH})$ and multifocal cortical superficial siderosis (cSS) in the left hemisphere. Multiple lobar cerebral microbleeds (CMBs) are also present, sometimes close to the cSS. (B) Patient with probable CAA with focal, bilateral cSS and lobar CMBs, in whom no ICHs were present. (C) Patient with a mixed pattern of bleeds (ICH and $\mathrm{CMBs}$ ) not fulfilling the Boston criteria for CAA: note the focal cSS in the left hemisphere (arrow) and the lobar CMBs in close vicinity to the cSS (arrowheads). FLAIR = fluid-attenuated inversion recovery; T2*-GRE $=\mathrm{T}^{*}$-weighted gradient recalled echo.

with patients without cSS (table 2). Patients with cSS more often had a history of transient focal neurologic episodes and chronic lobar ICH on MRI than patients without cSS. However, we found no association between the presence of cSS and acute lobar ICH, lobar CMBs, or white matter hyperintensity severity.
In univariable logistic regression analysis, factors associated with cSS were age, history of transient focal neurologic episodes, and chronic lobar ICH (table e-3). Hypertension showed a negative association with cSS. In multivariable logistic regression analysis, age, presence of chronic lobar $\mathrm{ICH}$, and history of transient focal neurologic episodes were independently associated with cSS, after adjusting for lobar CMBs (table 3). These results remained consistent after additional adjustment for hypertension and acute cSAH (data not shown). The results of cSS predictors in ordinal logistic regression (i.e., predictors of cSS extent: no cSS vs focal vs disseminated) were consistent and of similar effect size.

DISCUSSION To our knowledge, this multicenter retrospective study is the first systematic survey of the prevalence and clinical-radiologic associations of cSS in patients with probable sporadic CAA (diagnosed by the original Boston criteria) compared to those with ICH not fulfilling these criteria. A previous study investigated the presence of cSS in patients diagnosed with CAA on histopathology ${ }^{8}$ : cSS was detected in $60.5 \%$ of patients with histopathologically proven CAA ( $\mathrm{n}=38$; mean age 70 years) compared with none of the controls with histopathologically proven non-CAA ICH ( $\mathrm{n}=22$; mean age 54 years). ${ }^{8}$ The authors suggested that cSS might be helpful for the clinical diagnosis of CAA. ${ }^{8}$ cSS has also been found in patients with hereditary cerebral hemorrhage with amyloidosis-Dutch type, a distinct genetic type of CAA, and always in the direct vicinity of a lobar ICH or a CMB. ${ }^{19}$ Our findings strengthen the hypothesis that cSS (especially if disseminated) is a characteristic neuroimaging marker for CAA. We noted cSS in $40 \%$ of patients with probable CAA, disseminated cSS only in patients with probable CAA, and found that CSS is much rarer (prevalence less than 5\%) in patients with a "strictly deep" pattern of ICH than with probable CAA. We also detected cSS in $15 \%$ of patients with a single lobar ICH or mixed (lobar and deep) hemorrhages; however, in most of these patients with mixed hemorrhages, lobar CMBs were also present, suggesting that they might in fact harbor some degree of CAA pathology.

Among patients with a single lobar ICH and no CMBs (i.e., "possible CAA" according to the original Boston criteria ${ }^{14}$ ), the prevalence of cSS was also very low $(6 \%)$. In a validation of these criteria, 16 of 26 patients (62\%) classified as possible CAA had pathologically confirmed CAA, ${ }^{14}$ but only 11 of the pathologically diagnosed patients had T2*-GRE imaging, limiting the generalizability of the findings to current cohorts (including our study), in which such imaging is now routine. In another validation study of the Boston criteria, using T2*-weighted gradient echo MRI in a hereditary Dutch-type CAA population, all patients with lobar 
Table 2 Characteristics and comparison of CAA patients with and without cSS

\begin{tabular}{|c|c|c|c|c|}
\hline Characteristics & All probable CAA $(n=120)$ & $\operatorname{cSS}(+)(n=48)$ & $\operatorname{cSs}(-)(n=72)$ & p Value ${ }^{a}$ \\
\hline Age, y, mean $(95 \% \mathrm{Cl})$ & $71.8(70-73.5)$ & $74.8(72.3-77.3)$ & $69.7(67.4-72.1)$ & 0.005 \\
\hline Male, n (\%) & $67(55.8)$ & $25(52.1)$ & $42(58.3)$ & 0.499 \\
\hline Hypertension, $n(\%)^{b}$ & $72(64.3)$ & $20(46.5)$ & $52(75.4)$ & 0.002 \\
\hline Taking antithrombotics, $\mathrm{n}(\%)^{\mathrm{b}}$ & $25(22.1)$ & 9 (20.9) & 16 (22.9) & 0.811 \\
\hline History of symptomatic ICH, n (\%) & $46(40.7)$ & 18 (41.9) & $28(40)$ & 0.845 \\
\hline History of TFNE, $n(\%)$ & $26(21.7)$ & 19 (39.6) & $7(9.7)$ & $<0.001$ \\
\hline \multicolumn{5}{|l|}{ Neuroimaging characteristics } \\
\hline Chronic lobar ICH, n (\%) & 61 (51.3) & 31 (64.6) & $30(42.3)$ & 0.017 \\
\hline Acute lobar ICH, n (\%) & 57 (48.3) & $26(54.2)$ & 31 (44.3) & 0.291 \\
\hline Acute ischemic lesions, $n(\%)$ & $20(18.7)$ & $10(23.3)$ & $10(15.6)$ & 0.321 \\
\hline No. of CMBs, median (IQR) & $4(2-13.5)$ & $4.5(1-10.5)$ & $4(2-15.5)$ & 0.147 \\
\hline \multicolumn{5}{|l|}{ Lobar CMBs, n (\%) } \\
\hline 0 & $13(10.8)$ & 9 (18.8) & $4(5.6)$ & - \\
\hline 1 & $16(13.3)$ & $7(14.6)$ & 9 (12.5) & - \\
\hline $2-4$ & 34 (28.3) & $8(16.7)$ & $26(36.1)$ & - \\
\hline$\geq 5$ & $57(47.5)$ & $24(50)$ & $33(45.8)$ & - \\
\hline Acute cSAH, n (\%) & $15(12.5)$ & $9(18.8)$ & $6(8.3)$ & 0.091 \\
\hline Leukoaraiosis & & & & - \\
\hline Fazekas category, n (\%) & & & & - \\
\hline Absent & $19(16.1)$ & $8(17)$ & 11 (15.5) & $0.652^{c}$ \\
\hline Mild & $36(30.5)$ & 17 (36.2) & 19 (26.8) & - \\
\hline Moderate & 41 (34.8) & 15 (31.9) & 26 (35.6) & - \\
\hline Severe & 22 (18.6) & 7 (14.9) & 15 (21.1) & - \\
\hline Moderate to severe leukoaraiosis, $n(\%)$ & $63(53.4)$ & $22(46.8)$ & $41(57.6)$ & 0.244 \\
\hline
\end{tabular}

Abbreviations: $\mathrm{CAA}=$ cerebral amyloid angiopathy; $\mathrm{Cl}=$ confidence interval; $\mathrm{CMB}=$ cerebral microbleed; $\mathrm{cSAH}=$ convexity subarachnoid hemorrhage; $\mathrm{CSS}=$ cortical superficial siderosis; ICH = intracerebral hemorrhage; IQR = interquartile range; TFNE $=$ transient focal neurologic episodes.

${ }^{a}$ The $p$ values refer to differences between CAA patients with vs without cSS, using $x^{2}$ tests and the Fisher exact test for categorical variables, and 2-sample $t$ tests or Mann-Whitney $U$ tests depending on the distribution of continuous variables. ${ }^{b}$ There were 10 patients with missing data for one or more of these variables: hypertension $(n=9)$, taking antithrombotics $(\mathrm{n}=8)$, and history of symptomatic ICH $(\mathrm{n}=7)$.

${ }^{\mathrm{c}}$ The $\mathrm{p}$ value for trend.

ICH also had CMBs. ${ }^{19}$ Our data suggest that further pathologic correlation is required to determine the prevalence of pathologically confirmed CAA in patients with a single lobar $\mathrm{ICH}$ (and no $\mathrm{CMBs}$ or cSS) on

\begin{tabular}{|llr|}
\hline Table 3 & \multicolumn{2}{l}{$\begin{array}{l}\text { Multivariable regression analysis showing the factors associated with } \\
\text { cortical superficial siderosis in patients with probable cerebral amyloid } \\
\text { angiopathy }\end{array}$} \\
& OR (95\% Cl) & $p$ Value \\
Age & $1.09(1.03-1.15)$ & 0.002 \\
Lobar CMBs (for each category increase) & $0.77(0.50-1.21)$ & 0.260 \\
Chronic lobar ICH & $3.94(1.54-10.08)$ & 0.004 \\
History of transient focal neurologic episodes & $11.08(3.49-35.19)$ & $<0.001$ \\
\hline
\end{tabular}

Abbreviations: $\mathrm{Cl}=$ confidence interval; $\mathrm{CMB}=$ cerebral microbleed; $\mathrm{ICH}=$ intracerebral hemorrhage; $\mathrm{OR}=$ odds ratio.

${ }^{a}$ The model remains consistent when history of hypertension and acute convexity subarachnoid hemorrhage (\%) are included. appropriate blood-sensitive MRI sequences. Further studies with histopathologic confirmation or in vivo amyloid imaging $^{20}$ will also help to determine whether cSS is a useful diagnostic feature of CAA in patients with mixed patterns of hemorrhage.

In the healthy population-based Rotterdam Scan Study, cSS was found in $0.7 \%(7 / 1,062)$ of elderly individuals (mean age 69.6 years), all of whom had lobar CMBs (6 had strictly lobar CMBs, 5 of whom had multiple $\mathrm{CMBs}$ ) in close vicinity to the $\mathrm{CSS} .{ }^{10} \mathrm{In}$ agreement with our findings, the mean age of persons with cSS was higher than in those without CSS (mean age 79.9 vs 69.6 years; $p<0.001$ ), and also compared with persons who had CMBs but no cSS (mean age 71.8 years). ${ }^{10}$ Because the severity of CAA is age-related, ${ }^{1,3}$ these findings, together with our data showing an association of cSS with chronic ICH, suggest that $\mathrm{CSS}$ might be a marker 
of more advanced CAA, but this also requires pathologic confirmation, with standardized grading of CAA severity.

Experimental studies confirm that repeated bleeding into the subarachnoid space leads to subpial hemosiderin deposition. ${ }^{21}$ There are thus at least 2 possible pathophysiologic mechanisms that could lead to cSS deposition in CAA: a) repeated episodes of hemorrhage from brittle superficial cortical or leptomeningeal CAA-affected vessels into the subarachnoid space (independent of lobar $\mathrm{ICH}$ ); and b) leakage from a previous lobar ICH (or superficial lobar $\mathrm{CMBs}$ ) into the subarachnoid space. Our observation of cSS in 17 patients without chronic lobar $\mathrm{ICH}$, and even in those with chronic lobar ICH mostly distant from the ICH (in nearly $90 \%$ of cases in the contralateral hemisphere or bilaterally), favors a contribution from direct hemorrhage into the subarachnoid space, independent of lobar ICH. This implies that cSS may arise independently of known characteristic imaging features of CAA (lobar ICH and CMBs), supporting its role as an independent diagnostic marker; further studies are needed to confirm the value of cSS in improving the sensitivity of in vivo CAA diagnosis.

Perhaps surprisingly, we did not find any association between lobar CMBs and cSS, which may reflect selection bias toward generally advanced disease with high lobar CMBs prevalence in our cohort. Nevertheless, in some cases, cSS was observed close to one or more lobar CMBs (see, e.g., figure 2), suggesting that leakage from very superficial CMBs may also be a mechanism of cSS.

Another interpretation of the association between cSS and lobar ICH is that cSS precedes lobar ICH. Although our cross-sectional data cannot confirm this hypothesis, some recent data support this explanation. A retrospective study of 51 patients with cSS attributable to possible or probable CAA found that after a median 35.3 months' follow-up, $47.1 \%$ of the patients had new radiologic $\mathrm{ICH}$ or $\mathrm{CSAH}$, often at the site of pre-existing siderosis, ${ }^{13}$ providing preliminary evidence that cSS heralds a high risk of future $\mathrm{ICH}$. A small neuropathologic series of 6 autopsy cases of subcortical hematoma caused by CAA showed that at least in some cases the primary hemorrhage appeared to originate from the subarachnoid space. ${ }^{22}$ Further prospective studies are urgently required to determine the risk of future intracranial bleeding associated with cSS in CAA.

Our study confirms a strong independent association of cSS with transient focal neurologic episodes (sometimes called "amyloid spells"23), which are increasingly recognized in CAA and can resemble TIAs, migraine auras, or focal seizures. ${ }^{11,12,23}$ Such attacks could plausibly be caused by disruption of cortical function due to cSS, for example by superficial cortical hemosiderin deposition inducing focal seizures or cortical spreading depression. ${ }^{11,23-25}$ CAArelated transient focal neurologic episodes are associated with a high early risk of symptomatic lobar ICH (24.5\% [95\% CI: 15.8\%-36.9\%] at 8 weeks) $)^{11}$; cSS may be one mechanism underpinning this increased clinical risk, reflecting focally active CAA near the cortical surface. ${ }^{26}$

Our study has several strengths, including the systematic evaluation of MRI scans by trained raters using validated scales for a range of imaging markers of small-vessel disease. A limitation is the lack of pathologic confirmation of the CAA pathology. In view of the imperfect specificity of the Boston criteria, especially for the "possible CAA" category, ${ }^{14}$ we focused on patients fulfilling the criteria for probable CAA, for which the specificity is between $82 \%$ and $100 \% .^{14,19}$ Limitations of our study include the retrospective design, the variation in inception point of the disease at inclusion, and the potential of bias in our sample because MRIs were performed as part of routine clinical care, tending to exclude more severe cases of CAA and $\mathrm{ICH}$.

Our study indicates that cSS (particularly if disseminated) is a characteristic neuroimaging feature of CAA. Chronic lobar $\mathrm{ICH}$ is an independent risk factor for cSS, but the causal direction and mechanism of the association is uncertain: although leakage from previous lobar $\mathrm{ICH}$ into the subarachnoid space may lead to cSS, it is also possible that cSS heralds an increased risk of future lobar ICH. Further prospective studies are needed to clarify how cSS relates to future ICH risk, which may have important clinical relevance, for example regarding antithrombotic treatment. Our results also show that hemorrhage into the subarachnoid space, independent of lobar ICH, must also contribute to cSS in CAA, suggesting that cSS should be considered an additional neuroimaging marker of CAA-related hemorrhage.

\section{AUTHOR CONTRIBUTIONS}

A. Charidimou: project design, data collection, data analysis, write up. R.H. Jäger: imaging analysis advice, critical revisions. Z. Fox: data analysis, data interpretation, critical revisions. A. Peeters, Y. Vandermeeren, P. Laloux, and J.-C. Baron: data collection, critical revisions. D.J. Werring: project design, supervision, write up.

\section{ACKNOWLEDGMENT}

The authors thank Dr. Simone Gregoire for her assistance with establishing the multicenter cohort and for technical assistance with database organization.

\section{STUDY FUNDING}

Funded by the Stroke Association and the British Heart Foundation and supported by researchers at the National Institute for Health Research University College London Hospitals Biomedical Research Centre.

\section{DISCLOSURE}

A. Charidimou receives research support from the Greek State Scholarship Foundation, the Stroke Association, and the British Heart Foundation. R. Jäger has received research support from the Samantha Dickson Brain Tumour Trust and the Brain Research Trust. Z. Fox, A. Peeters, Y. Vandermeeren, P. Laloux, and J.-C. Baron report no disclosures. D. Werring receives research support from the Department of Health/Higher Education Funding Council for England (Clinical Senior Lectureship Award). Go to Neurology.org for full disclosures.

Received January 10, 2013. Accepted in final form May 3, 2013. 


\section{REFERENCES}

1. Charidimou A, Gang Q, Werring DJ. Sporadic cerebral amyloid angiopathy revisited: recent insights into pathophysiology and clinical spectrum. J Neurol Neurosurg Psychiatry 2012; 83:124-137

2. Viswanathan A, Greenberg SM. Cerebral amyloid angiopathy in the elderly. Ann Neurol 2011;70:871-880.

3. Vinters HV. Cerebral amyloid angiopathy: a critical review. Stroke 1987;18:311-324.

4. Greenberg SM, Vernooij MW, Cordonnier C, et al. Cerebral microbleeds: a guide to detection and interpretation. Lancet Neurol 2009;8:165-174.

5. Raposo N, Viguier A, Cuvinciuc V, et al. Cortical subarachnoid haemorrhage in the elderly: a recurrent event probably related to cerebral amyloid angiopathy. Eur J Neurol 2011;18:597-603.

6. Beitzke M, Gattringer T, Enzinger C, Wagner G, Niederkorn K, Fazekas F. Clinical presentation, etiology, and long-term prognosis in patients with nontraumatic convexal subarachnoid hemorrhage. Stroke 2011;42:3055-3060.

7. Kumar S, Goddeau RP, Selim MH, et al. Atraumatic convexal subarachnoid hemorrhage: clinical presentation, imaging patterns, and etiologies. Neurology 2010;74: 893-899.

8. Linn J, Halpin A, Demaerel P, et al. Prevalence of superficial siderosis in patients with cerebral amyloid angiopathy. Neurology 2010;74:1346-1350.

9. Linn J, Herms J, Dichgans M, et al. Subarachnoid hemosiderosis and superficial cortical hemosiderosis in cerebral amyloid angiopathy. AJNR Am J Neuroradiol 2008;29: 184-186.

10. Vernooij MW, Ikram MA, Hofman A, Krestin GP, Breteler MM, van der Lugt A. Superficial siderosis in the general population. Neurology 2009;73:202-205.

11. Charidimou A, Peeters A, Fox Z, et al. Spectrum of transient focal neurological episodes in cerebral amyloid angiopathy: multicentre magnetic resonance imaging cohort study and meta-analysis. Stroke 2012;43:2324-2330.

12. Greenberg SM, Vonsattel JP, Stakes JW, Gruber M, Finklestein SP. The clinical spectrum of cerebral amyloid angiopathy: presentations without lobar hemorrhage. Neurology 1993;43:2073-2079.

13. Linn J, Wollenweber FA, Lummel N, et al. Superficia siderosis is a warning sign for future intracranial hemorrhage. J Neurol 2012;260:176-181.
14. Knudsen KA, Rosand J, Karluk D, Greenberg SM. Clinical diagnosis of cerebral amyloid angiopathy: validation of the Boston criteria. Neurology 2001;56:537-539.

15. Greenberg SM, Nandigam RN, Delgado P, et al. Microbleeds versus macrobleeds: evidence for distinct entities. Stroke 2009;40:2382-2386.

16. Gregoire SM, Chaudhary UJ, Brown MM, et al. The Microbleed Anatomical Rating Scale (MARS): reliability of a tool to map brain microbleeds. Neurology 2009;73:1759-1766.

17. Gregoire SM, Charidimou A, Gadapa N, et al. Acute ischaemic brain lesions in intracerebral haemorrhage: multicentre cross-sectional magnetic resonance imaging study. Brain 2011;134:2376-2386.

18. Inzitari D, Simoni M, Pracucci G, et al. Risk of rapid global functional decline in elderly patients with severe cerebral age-related white matter changes: the LADIS Study. Arch Intern Med 2007;167:81-88.

19. van Rooden S, van der Grond J, van den Boom R, et al. Descriptive analysis of the Boston criteria applied to a Dutch-type cerebral amyloid angiopathy population. Stroke 2009;40:3022-3027.

20. Dierksen GA, Skehan ME, Khan MA, et al. Spatial relation between microbleeds and amyloid deposits in amyloid angiopathy. Ann Neurol 2010;68:545-548.

21. Koeppen AH, Dickson AC, Chu RC, Thach RE. The pathogenesis of superficial siderosis of the central nervous system. Ann Neurol 1993;34:646-653.

22. Takeda S, Yamazaki K, Miyakawa T, et al. Subcortical hematoma caused by cerebral amyloid angiopathy: does the first evidence of hemorrhage occur in the subarachnoid space? Neuropathology 2003;23:254-261.

23. Charidimou A, Law R, Werring DJ. Amyloid "spells" trouble. Lancet 2012;380:1620.

24. Dreier JP. The role of spreading depression, spreading depolarization and spreading ischemia in neurological disease. Nat Med 2011;17:439-447.

25. Dreier JP, Ebert N, Priller J, et al. Products of hemolysis in the subarachnoid space inducing spreading ischemia in the cortex and focal necrosis in rats: a model for delayed ischemic neurological deficits after subarachnoid hemorrhage? J Neurosurg 2000;93:658-666.

26. Charidimou A, Baron JC, Werring DJ. Transient focal neurological episodes, cerebral amyloid angiopathy, and intracerebral hemorrhage risk: looking beyond TIAs. Int J Stroke 2013;8:105-108.

\section{Save These Dates for AAN CME Opportunities!}

Mark these dates on your calendar for exciting continuing education opportunities, where you can catch up on the latest neurology information.

\section{Regional Conference}

- October 25-27, 2013, Las Vegas, Nevada, Encore at Wynn Hotel

\section{AAN Annual Meeting}

- April 26-May 3, 2014, Philadelphia, Pennsylvania, Pennsylvania Convention Center 


\section{Neurology}

\section{Prevalence and mechanisms of cortical superficial siderosis in cerebral amyloid angiopathy \\ Andreas Charidimou, Rolf Hans Jäger, Zoe Fox, et al.}

Neurology 2013;81;626-632 Published Online before print July 17, 2013

DOI 10.1212/WNL.0b013e3182a08f2c

\section{This information is current as of July 17, 2013}

\section{Updated Information \& Services}

\section{Supplementary Material}

\section{References}

\section{Citations}

\section{Subspecialty Collections}

\section{Permissions \& Licensing}

Reprints including high resolution figures, can be found at: http://n.neurology.org/content/81/7/626.full

Supplementary material can be found at: http://n.neurology.org/content/suppl/2013/07/18/WNL.0b013e3182a08 f2c.DC1

This article cites 26 articles, 13 of which you can access for free at: http://n.neurology.org/content/81/7/626.full\#ref-list-1

This article has been cited by 16 HighWire-hosted articles: http://n.neurology.org/content/81/7/626.full\#\#otherarticles

This article, along with others on similar topics, appears in the following collection(s):

\section{All Cerebrovascular disease/Stroke}

http://n.neurology.org/cgi/collection/all_cerebrovascular_disease_strok e

Intracerebral hemorrhage

http://n.neurology.org/cgi/collection/intracerebral_hemorrhage

MRI

http://n.neurology.org/cgi/collection/mri

Information about reproducing this article in parts (figures,tables) or in its entirety can be found online at:

http://www.neurology.org/about/about_the_journal\#permissions

Information about ordering reprints can be found online:

http://n.neurology.org/subscribers/advertise

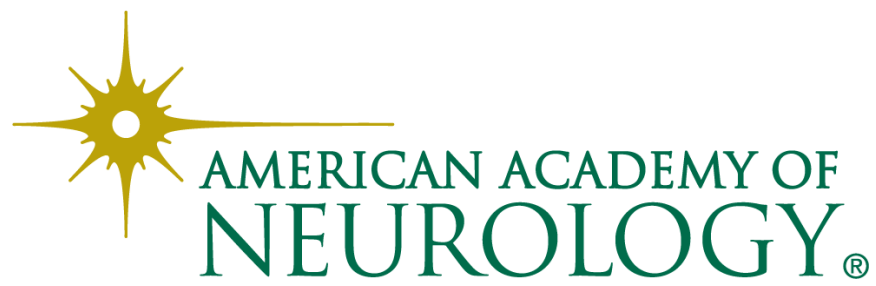

